\title{
Pintar COM LUZ: O esmalte AZUL NA PINTURA de VITRAL
}

\author{
Márcia Vilarigues* e Andreia Machado \\ Departamento de Conservação e Restauro - Unidade de Investigação VICARTE - Vidro e Cerâmica para as Artes \\ Faculdade de Ciências e Tecnologia, Universidade Nova de Lisboa \\ mgv@fct.unl.pt
}

\begin{abstract}
Painting with light: blue enamels in stained glass painting - The painting applied on stained glass presents a challenge for conservation-restoration due to its fragility. Modern materials used for stained glass painting do not reflect the old technologies and raw materials and therefore cannot be used as reference materials in studies of mechanisms of degradation and implementation of conservation measures. In this context, knowledge of historical painting techniques is fundamental for a better understanding of the different mechanisms that lead to their degradation and an opportunity to better understand the history of this artistic discipline.
\end{abstract}

\begin{abstract}
A pintura sobre vitral apresenta um desafio para a conservação-restauro dada a sua fragilidade. Os materiais modernos utilizados para a pintura do vitral não reflectem as antigas tecnologias e matérias primas e por esse motivo não podem ser utilizados como materiais de referência em estudos de mecanismos de degradação e de implementação de medidas de conservação. Neste contexto, o conhecimento das técnicas de pintura histórica revela-se fundamental para uma melhor compreensão dos diferentes mecanismos que levam à sua degradação e constitui ainda uma oportunidade de melhor compreender a história desta disciplina artística.
\end{abstract}

\section{INTRODUÇÃO}

Um conhecimento detalhado dos métodos e materiais utilizados pelos artistas é essencial para desvendar técnicas de produção artística e para colocar obras no seu contexto histórico. Estes estudos abrem ainda a possibilidade da redescoberta de diversos materiais e técnicas quer por conservadores e historiadores, quer por artistas.

O estudo das técnicas de produção artística tem várias fontes possíveis, sendo uma delas a reconstrução seguindo receitas e procedimentos descritos em documentos históricos sobre técnicas artísticas. As reconstruções permitem-nos ter uma maior compreensão do objecto original e do contexto em que foi criado, e são de grande importância no estudo dos mecanismos de degradação dos materiais, sendo este conhecimento essencial para definir procedimentos de conservação mais adequados.

Para desvendar as técnicas de produção utilizadas por artistas e fabricantes de vidro medievais é necessário uma ligação estreita entre as fontes escritas e a experimentação laboratorial. Neste contexto pretendemos neste estudo reconstruir esmalte azul de acordo com as receitas e indicações de Antonio Neri publicadas na sua obra l'Arte Vetraria (1612), obra de referência para quase todas as publicações dos séculos XVII e XVIII relativas a este tema [1]. O esmalte azul histórico aplicado sobre vidro na produção de vitrais apresenta diversos sinais de degradação e espera-se assim um maior entendimento sobre estes sinais de forma a projectar medidas de conservação apropriadas.

\section{O VITRAL}

"A sala resplandece iluminada ao centro. Resplandece de facto o que está distintamente unido àquilo que ilumina e eis que uma luz nova inunda, brilha como nobre obra."

Suger, "De rebus in adm. sua gestis”, PL 186, col.1229 [2]

$\mathrm{O}$ vidro, como meio que estabelece a união entre a luz e o espaço interior, tem o seu expoente máximo no vitral, onde é explorada a vivacidade da cor ligada ao fulgor da luz que a atravessa. Desta interacção resultam jogos de cores elementares, com regiões de claro-escuro, que geram luminosidade a partir da composição de conjunto.

O primeiro passo de idealização do vitral era realizado sobre uma prancha de madeira: a tábua de vitralista, a qual foi posteriormente substituída por cartão. Aí, são marcados os limites do vitral, as linhas de corte da composição e, por fim, delineados os elementos para o posterior trabalho de pintura. O vidro é então cortado em pedaços com as formas e cores indicadas no desenho original, sendo depois pintado em diferentes fases dependendo dos detalhes, tonalidades e cores definidos. Por fim, os elementos de vidro (pintados ou não) são montados, tradicionalmente, em calha do chumbo [3].

\section{MATERIAIS DE PINTURA DO VITRAL}

A substância mais amplamente utilizada para a pintura do vitral, sendo também a primeira, é a grisalha, composta por 
uma mistura de óxidos metálicos (ferro e/ou cobre) e fundente (vidro e óxido de chumbo) a qual era aglutinada com goma-arábica, vinho ou urina. É então aplicada, por regra no anverso do painel (i.e. na superfície do painel virada para o interior do edifício). Depois de seca, a grisalha é levada a cozer em pequenos fornos a temperaturas entre $600{ }^{\circ} \mathrm{C} \mathrm{e}$ $750{ }^{\circ} \mathrm{C}$. Deste modo é obtida a adesão do fundente com os pigmentos em suspensão e a superfície do vidro, formando-se uma camada de tinta $[3,4]$.

A partir de princípios do séc. XIV, passou também a ser possível pintar com amarelo, fazendo uso de sais de prata. O processo de obtenção e aplicação do amarelo de prata, foi descrito pela primeira vez por Antonio de Pisa [5]. Este é aplicado no reverso (a superfície virada para o exterior do edifício) de vidros incolores para realce de pormenores anatómicos, adereços, etc. O amarelo de prata era preparado com sais de prata frequentemente misturados com ocre ou argilas. O vidro pintado com esta mistura é levado ao forno e cozido a uma temperatura inferior àquela a que eram submetidas as grisalhas, motivo pelo qual era cozido separadamente e depois destas, evitando o risco de sobrecozedura. Durante a cozedura, opera-se a redução do sal de prata a prata metálica, dispersando-se esta na superfície do vidro sob a forma de colóides [6].

O uso de esmaltes (pigmentos feitos de vidro colorido moído) só passou a ser prática no vitral a partir do início do século XVI [1]. A introdução dos esmaltes na arte do vitral constituiu uma inovação artística, permitindo um aumento na gama cromática utilizada para colorir os painéis, assim como a existência de diversas cores numa única peça de vidro. A paleta completa de cores foi sendo progressivamente introduzida ao longo de meio século, primeiro o azul, seguido do violeta, castanho, vermelho carnação e por último o verde [7].

O esmalte é composto por um vidro base com um agente colorante (óxidos de iões metálicos), fundindo a uma temperatura mais baixa do que a temperatura de fusão do vidro sobre o qual é aplicado [1,7].

\section{Os TRATADOS DE PINTURA SOBRE VIDRO}

Durante a Idade Média e o Renascimento foi publicado um número significativo de textos de carácter técnico relativos à produção artística. O interesse por estes textos remonta ao final do século XIII, quando foi pela primeira vez traduzido o tratado do monge Theophilus, intitulado De Diversis Artibus, escrito durante o século XII [8].

O tratado De Diversis Artibus, assim como A Memória de António de Pisa são, os tratados mais significativos acerca da arte e da técnica do vitral no século XIV. Nos séculos seguintes muitos outros manuscritos foram publicados e traduzidos, salientando-se o tratado de António Neri, L'Arte Vetraria (1612) e o de André Felibien, Les principles de l'Architecture, de la Sculpture, de la Peinture et dês Autres Arts qui en dependant (1676). O tratado original de Neri foi traduzido para o latim pelo inglês Christopher Merret
(1662) e para o alemão pelo químico Johann Friedrich Kunckel (1679) [9].

Durante o século XVIII surgiram diversas traduções, com anotações e novas receitas, de acordo com as novas tecnologias disponíveis. Um exemplo importante é o tratado de Pierre Le Vieil, L'Art de la Peinture sur verre et de la Vitrerie (1774). O tratado é dividido em várias partes, sendo a primeira dedicada à origem do vidro, sua antiguidade e utilidade desde tempos antigos, incluindo a pintura e os vidros das igrejas, entre outros temas similares. A segunda parte do tratado é dedicada às receitas tanto para a fabricação da pasta do vidro, como também à forma de fazer esmaltes e pintura sobre vidro. Pierre Le Vieil acrescenta comentários à edição inglesa de Robert Dossie The handmaid to the Arts (1758), obra não traduzida para o francês, na qual são discutidos os trabalhos de Neri e as traduções destes tratados, Merret e Kunckel. O tratado de Pierre Le Vieil inclui também traduções para francês desses mesmos autores, assim como de Andre Felibien.

Já no século XIX destaca-se o Nouveau Manuel complet de la peinture sur verre sur porcelaine et sur émail, escrito por Reboulleau (1825).

O Esmalte Azul no tratado de António Neri: matérias-PRIMAS E RECEITAS

Tal como descrito anteriormente o esmalte é um vidro colorido que funde a uma temperatura inferior à do vidro que constitui o substrato no qual é aplicado. O vidro fundido é arrefecido rapidamente e reduzido a um pó fino por moagem. Este pó é então misturado com um aglutinante, geralmente aquoso, para se obter uma tinta que possa ser aplicada sobre o vidro. Após a secagem, o painel de vidro pintado é recozido e o pó funde, formando uma fina camada colorida sobre o substrato de vidro.

Os esmaltes podem apresentar diversos problemas de conservação, observando-se frequentemente microfissuras, má adesão ao substrato e destacamentos, sendo que o esmalte azul, parece ser aquele mais susceptível à degradação $[1,9]$ (ver Figura 1). Neste sentido, torna-se de grande relevância estudar as formas de preparação do esmalte azul e sua aplicação, de forma a poder planear medidas que apoiem medidas de preservação.

Os esmaltes azuis devem a sua cor à presença de cobalto na sua composição. De acordo com a literatura o cobalto utilizado era exportado das minas de Schneeberg na Saxónia, que detinham o monopólio do comércio do cobalto durante o século XVII [10].

A produção do esmalte azul descrita nos diversos tratados tem como base as receitas do tratado original de António Neri [11]. No livro sexto do seu tratado, António Neri divide a produção dos esmaltes em duas etapas: a produção do esmalte base (Materia com la quale si fanno tutti li Smalti, Cap. XCIII) e a produção de cada uma das cores. Para o esmalte azul existem três receitas, associadas a diferentes 

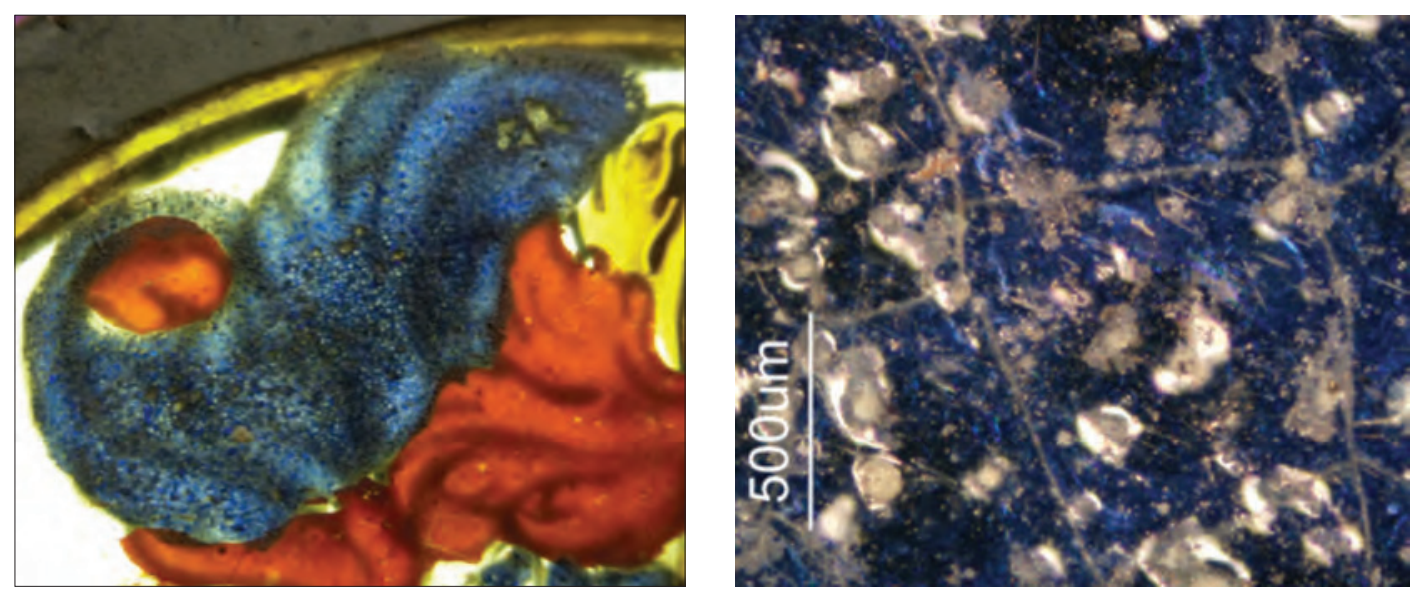

Figura 1 - Fotografia de detalhe (a) e imagem de microscopia óptica (b) de um fragmento de esmalte azul aplicado num vitral datado do século XVII, apresentando sinais de degradação (Créditos fotográficos: Fernanda Barroso)

tonalidades: dois turquesa (Smalto Turchino, Cap. XCV) e um "outro azul” (Altro Smalto azzurro, Cap. XCVI).

Na tabela 1 apresentam-se as matérias-primas indicadas por Antonio Neri, assim como a sua fórmula química correspondente.

Tabela 1 - Matérias-primas e fórmula química correspondente dos compostos necessários para a produção de um esmalte azul de acordo com as receitas de António Neri para a produção de um Altro Smalto azzurro

\begin{tabular}{|c|c|c|c|}
\hline & & Matéria-Prima & $\begin{array}{l}\text { Fórmula } \\
\text { química }\end{array}$ \\
\hline \multirow{4}{*}{ Esmalte base } & \multirow{2}{*}{ Frita de Cristallo } & $\begin{array}{l}\text { Tarso } \\
\text { (Seixos do Rio Ticino) }\end{array}$ & $\mathrm{SiO}_{2}$ \\
\hline & & $\begin{array}{l}\text { Sal de Polverino } \\
\text { (cinzas de plantas costeiras) }\end{array}$ & $\mathrm{Na}_{2} \mathrm{CO}_{3}$ \\
\hline & & $\begin{array}{l}\text { Sal di Tártaro } \\
\text { (cinzas de borras de vinho) }\end{array}$ & $\mathrm{K}_{2} \mathrm{CO}_{3}$ \\
\hline & & chumbo e estanho calcinados & $\mathrm{Pb}_{2} \mathrm{SnO}_{4}$ \\
\hline \multirow{3}{*}{ Esmalte Azul } & & Zaffera & $\mathrm{CoO}$ \\
\hline & & $\begin{array}{l}\text { Ramina di tre cotte } \\
\text { ou }\end{array}$ & $\mathrm{CuO}$ \\
\hline & & Manganês de Piemonte & $\mathrm{MnO}$ \\
\hline
\end{tabular}

Os seixos do Rio Ticino escolhidos por Neri para a produção da frita de cristallo são muito puros em quartzo, - "sem veios e que não apresente a aparência amarela da ferrugem" - tal como se pode verificar das análises realizadas por ICP-MS (ver tabela 2) de amostras destes seixos fornecidas pelo Departamento de Ambiente e Ciências da Terra da Universidade de Pavia.

Tabela 2 - Análise por ICP-MS dos seixos do rio Ticino (\%m/m)

\begin{tabular}{c|c|c|c|c}
\hline $\mathrm{SiO}_{2}$ & $\mathbf{A l}_{2} \mathbf{O}_{3}$ & $\mathbf{C a O}$ & $\mathbf{M n O}$ & $\mathbf{T i O}_{2}$ \\
\hline 99,9 & 0,03 & 0,04 & 0,003 & 0,003 \\
\hline
\end{tabular}

As cinzas de plantas costeiras para a produção do sal de polverino utilizadas provinham da região do Levante, ou da Síria, e eram consideradas por Neri como sendo melhores do que a "Soda di Spagna". No seu tratado Neri descreve ainda a preparação e purificação destas cinzas de forma a obter o sal que será depois misturado com o tarso moído finamente - "moagem tem de ser muito bem feita, de maneira que fique como farinha”. O processo da fusão desta mistura, num forno, deveria ser feito de forma muito cuidadosa de forma a obter uma frita "branca como a neve do céu".

A calcinação dos metais chumbo e estanho deveria ser feita num forno não muito quente, aproximadamente à temperatura de fusão dos dois metais $\left(327,4{ }^{\circ} \mathrm{C}\right.$ para o chumbo e $232{ }^{\circ} \mathrm{C}$ para o $\mathrm{Sn}$ ) [12], obtendo-se no final do processo um pó amarelo.

Entre os séculos XV e XVIII a fonte de cobalto utilizada no fabrico do esmalte azul eram minérios provenientes das minas de Schneeberg (Erzgebirge), em particular a Skutterudite, um arseniato de cobalto com níquel e bismuto e com quantidades variáveis de impurezas [13].

Neste trabalho amostras do minério skutterudite, cedidas pela Universidade de Freiberg, foram analisadas pelas técnicas de difracção de raios- $X$ e fluorescência de raios- $X$ de energia dispersiva (Figuras 2 e 3). No difractograma apresentado na Figura 2 é bem visível a presença do arseniato de cobalto, assim como a presença de quartzo e óxido de bismuto. Já no espectro de fluorescência de raios-X é visível a presença de níquel e de ferro.

No seu tratado, Antonio Neri refere a também preparação deste minério, com o objectivo de purificá-lo. O procedimento inclui a sua calcinação e lavagem com vinagre e 
água quente, seguidas de decantação e secagem. O processo de calcinação do minério leva a uma diminuição acentuada do conteúdo de arsénio, como é visível no espectro de fluorescência de raios-X (Figura 3).

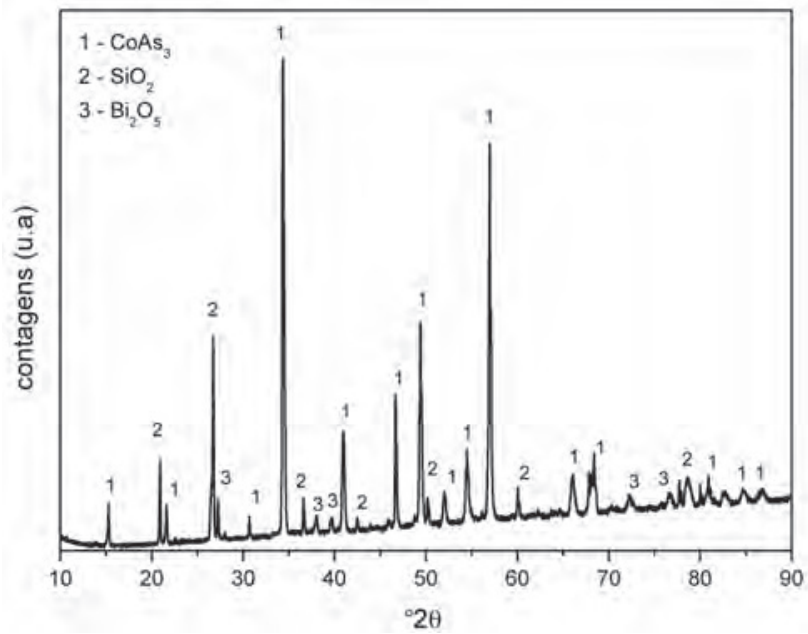

Figura 2 - Espectro de difracção de raios-X do minério skutterudite proveniente das minas de Schneeberg (Erzgebirge), Alemanha

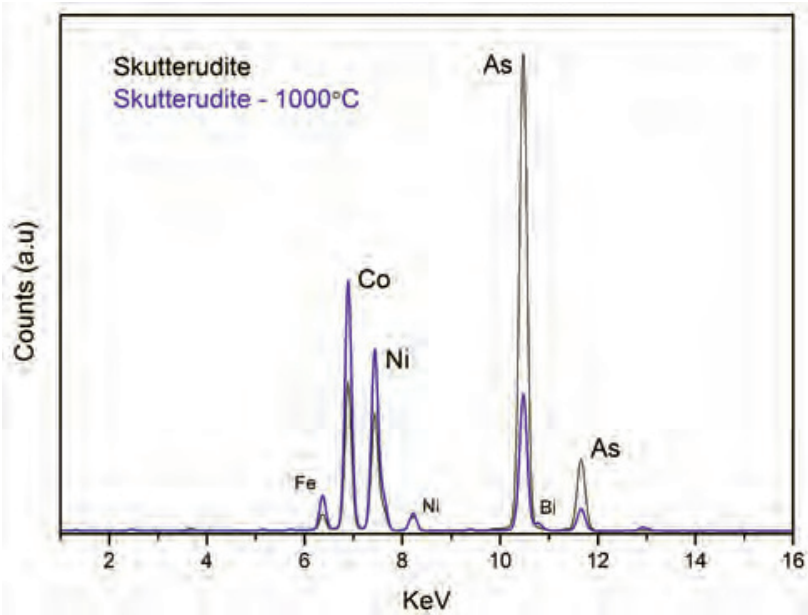

Figura 3 - Espectro de fluorescência de raios-X do minério skutterudite antes e após calcinação a $1000^{\circ} \mathrm{C}$

A produção do esmalte azul consistia então na mistura do esmalte base com o zaffre e ainda com adição de manganês ou cobre calcinados permitindo a obtenção de diferentes tonalidades do azul.

\section{AS REPRODUÇÕES DAS RECEITAS}

A informação obtida do estudo do tratado de António Neri serviu de base para a experimentação da produção de reproduções de esmaltes azuis. Os esmaltes foram produzidos com ingredientes puros e fundidos em fornos eléctricos modernos. É por isso importante ter em mente que as matérias-primas e condições de produção utilizadas são diferentes da realidade histórica.

Tendo como ponto de partida as receitas descritas por António Neri, foi concebida uma receita teórica simplificada, da qual foram retiradas todas as "impurezas” associadas às matérias-primas originais (Tabela 3).
A mistura dos ingredientes puros foi colocada num cadinho de alumina e levada a uma temperatura de $1200{ }^{\circ} \mathrm{C}$ durante 10 horas. O vidro fundido foi vertido para água fria, levando a que devido ao arrefecimento rápido se quebrasse em pedaços. O vidro obtido foi então seco e moído em moinho de pilão de ágata. Obteve-se assim um fino pó pronto a ser aplicado sobre o vidro.

\section{A APLICAÇÃO DO ESMALTE AZUL SOBRE VIDRO}

António Neri não descreve no seu tratado a forma de aplicação dos esmaltes sobre o vidro. Para obtermos essa informação foi necessário explorar tratados posteriores, em particular The handmaid to the Arts de Robert Dossie [14]. Neste tratado o autor refere que o pó de esmalte pode ser aplicado sobre uma superfície (vidro ou outra) de duas formas. Na primeira o pigmento é misturado com um ligante, de preferência óleo essencial de lavanda, formando-se uma pasta a ser aplicada com pincel. No entanto, desta forma pode ser difícil obter uma camada homogénea. A melhor forma de se obter esta camada será aplicando o óleo sobre a superfície a ser pintada, dispersando depois o pó sobre o óleo, retirando-se finalmente todo o esmalte que não fique bem agarrado à superfície. Em ambos os casos, o autor chama a atenção para que o tempo de secagem antes do recozimento do esmalte deve ser curto.

Na Figura 4 apresentam-se os resultados obtidos após a aplicação do esmalte sobre vidro utilizando as duas técnicas e posterior recozimento a $700{ }^{\circ} \mathrm{C}$ durante 10 minutos. Como se pode observar o segundo método permite obter uma camada muito mais homogénea e semelhante ao que podemos encontrar em vitrais históricos.
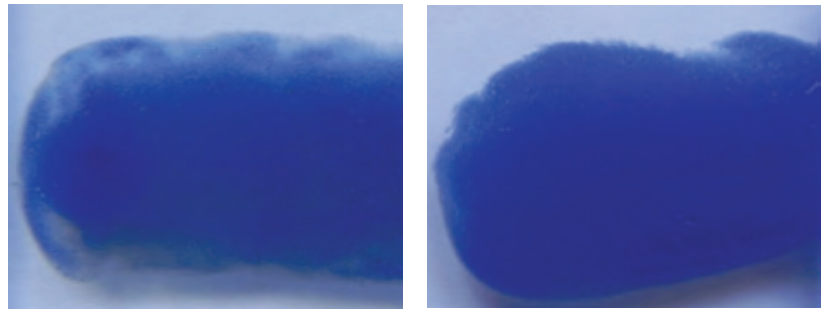

Figura 4 - Fotografia de detalhe da aplicação (a) com pincel de esmalte misturado com óleo de lavanda (b) de esmalte disperso sobre óleo de lavanda aplicado sobre vidro

\section{EM JEITO DE CONCLUSÃO}

Não podemos esquecer que receitas escritas num dado período, num dado local, podem não ser representativas das práticas desse período e desse local, sendo por isso fundamental confrontar os resultados das reproduções com o das obras de arte. As reconstruções históricas realizadas são um material de grande importância para os futuros estudos de degradação dos esmaltes azuis e para comparação com casos reais de pintura em vitral. Esperamos deste modo contribuir para a preservação deste património para esta geração e para as gerações futuras. 
Tabela 3 - Matérias-primas e compostos químicos associados utilizados para a produção de um esmalte azul de acordo com as receitas de António Neri para a produção de um Altro Smalto azzurro

\begin{tabular}{|c|c|c|c|}
\hline \multirow{11}{*}{ Esmalte base } & \multirow{11}{*}{ Frita de Cristallo } & Tarso & $\mathrm{SiO}_{2}(\% \mathrm{~m} / \mathrm{m})$ \\
\hline & & $50 \mathrm{lb}$ & 28,6 \\
\hline & & & \\
\hline & & Sal de Polverino & $\mathrm{Na}_{2} \mathrm{CO}_{3}(\% \mathrm{~m} / \mathrm{m})$ \\
\hline & & $130 \mathrm{lb}$ & 18,6 \\
\hline & & Sal di Tártaro & $\mathrm{K}_{2} \mathrm{CO}_{3}(\% \mathrm{~m} / \mathrm{m})$ \\
\hline & & $8 \mathrm{oz}$ & 0,6 \\
\hline & & Chumbo calcinado & $\mathrm{PbO}(\% \mathrm{~m} / \mathrm{m})$ \\
\hline & & $30 \mathrm{lb}$ & 22,5 \\
\hline & & Estanho calcinado & $\mathrm{SnO}_{2}(\% \mathrm{~m} / \mathrm{m})$ \\
\hline & & $33 \mathrm{lb}$ & 24,7 \\
\hline \multirow{4}{*}{ Esmalte Azul } & & Zaffera & $\mathrm{CoO}(\% \mathrm{~m} / \mathrm{m})$ \\
\hline & & $2 \mathrm{oz}(1,16 \mathrm{lb})$ & 4,8 \\
\hline & & Ramina di tre cotte & $\mathrm{CuO}(\% \mathrm{~m} / \mathrm{m})$ \\
\hline & & 2 pwt (0,006 lb) & 0,2 \\
\hline
\end{tabular}

\section{AgradeCIMENTOS}

Este trabalho foi desenvolvido no âmbito do projecto de investigação: A colecção de vitrais de D. Fernando II Montando o puzzle (PTDC/EPH-PAT/3579/2012 - ), e do projecto de doutoramento com a referência SFRH/ $\mathrm{BD} / 78882 / 2011$

\section{REFERÊNCIAS}

[1] O. Schalm et al., Spectrochimica Acta B 64 (2009) 812$-820$

[2] U. Eco, “Arte e Beleza na Estética Medieval”, Editorial Presença, 2000

[3] P. Redol, "O Mosteiro da Batalha e o vitral em Portugal nos séculos XV e XVI”, Câmara Municipal da Batalha, 2003

[4] M. Verità, Grisaille, Jaune d'argent, Sanguine, Émail e Peinture à Froid (1996) 61-68

[5] C. Lautier, D. Sandron, «Antoine de Pise - L'art du vitrail vers 1400», Editions CTHS, 2008

[6] D. Jembrih-Simburger et al., J. Anal. Atom. Spectrom. 17 (2002) 321-328

[7] L. Cannon, "Stained Glass in the Burrell Collection", Chambers, 1991

[8] K. Boulanger, Bibliothèque de l'École des chartes 162 (2004) 9-33

[9] J. Caen, "The Production of Stained Glass in the country of Flanders and the duchy of Brabant from the $\mathrm{XV}^{\text {th }}$ to the XVIII ${ }^{\text {th }}$ centuries: materials and techniques” Brepols, Antuérpia 2009

[10] Holbach, «Art de la Verrerie, de Neri, Merret et Kunckel», Paris, 1752

[11] A. Neri, "L’Arte Vetraria”, Florença, 1612

[12] N. Glinka, “Química Geral”, Editora Mir, Moscovo, 1988

[13] B. Gratuze et al., Revue d’Archéometrie 20 (1996) 97-108

[14] R. Dossie, «Handmaid to the Arts», Londres, 1758
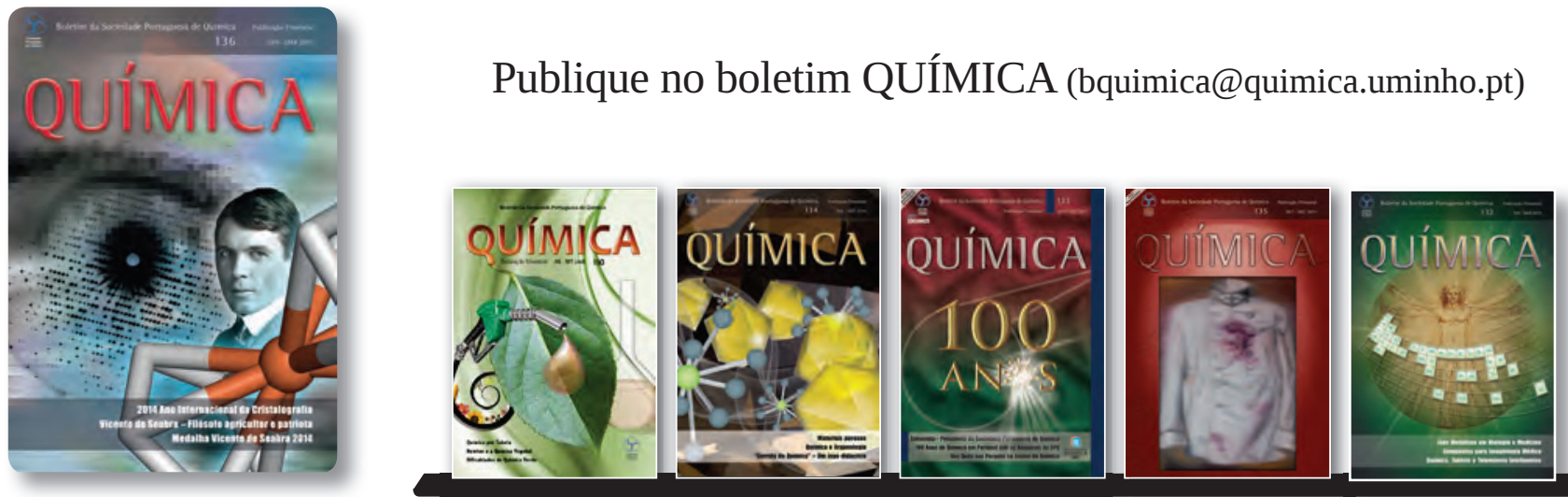


\section{Antoine-Laurent LAVOISIER}

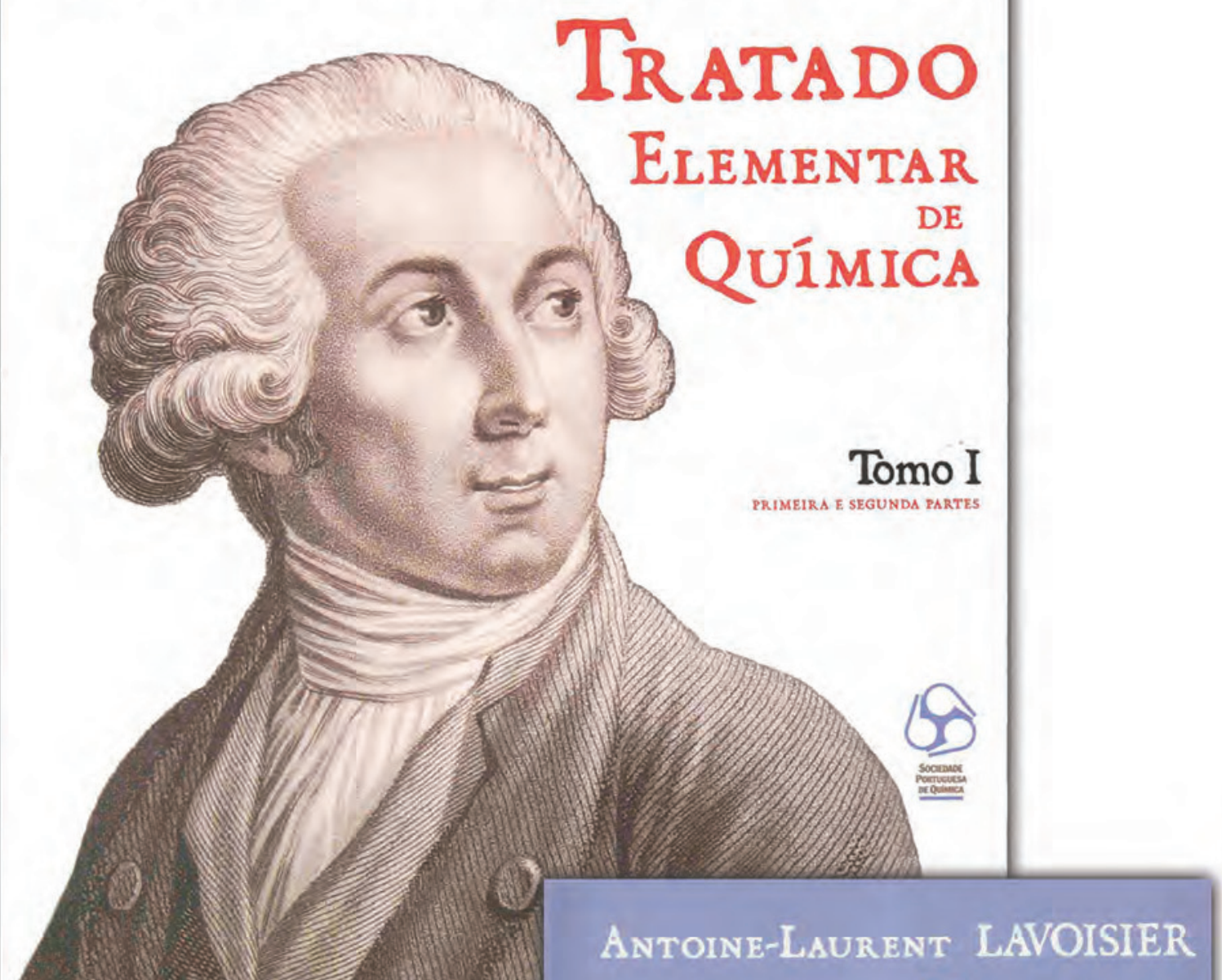

0 "Tratado Elementar de Química» de Antoine-Laurent Lavoisier (1743-1794), publicado em Paris em 1789, é um dos grandes clássicos da Química. Escrito na nova nomenclatura proposta também por Lavoisier, em conjunto com Morveau, Berthollet e Fourcroy, é a primeira obra de química moderna, abandonando-se as inadequadas designações de raízes alquímicas e a desacreditada teoria do flogisto. No tratado, ilustrado pela mulher do autor, Marie-Anne-Pierrette Paulze Lavoisier, formula-se de forma clara a conservação da matéria e define-se elemento químico de forma operacional.

No centenário da fundação da Sociedade Portuguesa de Química, publica-se finalmente uma cuidada tradução portuguesa do "Traité», modernizada e anotada.

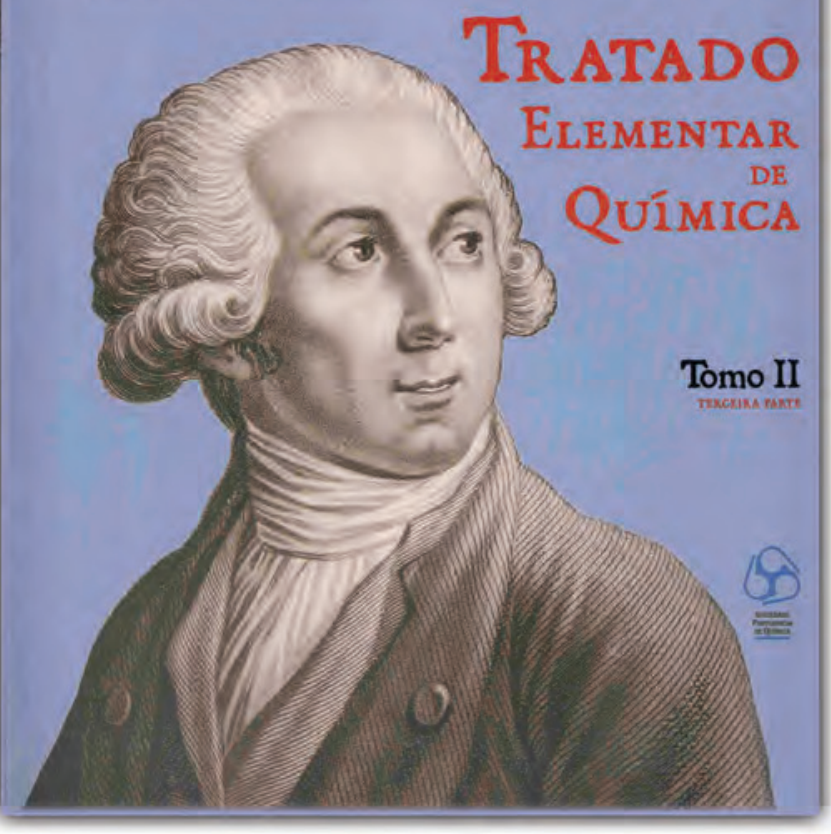

